\title{
Anti-inflammatory dietary intervention improved metabolic profile and body composition of obese adults
}

\author{
Gordana Kenđel Jovanović ${ }^{1}$, Sandra Pavičić Žeželj ${ }^{1,2}$, Sanja Klobučar Majanović ${ }^{3,2}$, \\ Ines Mrakovčić Sutić ${ }^{2}$ and Greta Krešić \\ ${ }^{1}$ Teaching Institute of Public Health of Primorsko-goranska County, Rijeka, Croatia, \\ ${ }^{2}$ University of Rijeka, Faculty of Medicine, Rijeka, Croatia, \\ ${ }^{3}$ Clinical Hospital Centre Rijeka, Rijeka, Croatia and \\ ${ }^{4}$ University of Rijeka, Faculty of Tourism and Hospitality Management, Rijeka, Croatia
}

\begin{abstract}
It has become evident that chronic low-grade inflammation associated with excess adipose tissue plays an important role in the etiology of insulin resistance and other obesity related metabolic disturbances. Weight loss intervention focusing on anti-inflammatory dietary modification might help to attenuate and eventually reverse these disturbances. In this random case-control nutrition intervention study we hypothesized that obese patients will improve their inflammatory and metabolic status together with body composition following anti-inflammatory diet during 24 weeks. Body composition parameters of 42 obese patients, who completed the study, were determined by bioelectrical impedance analysis (Seca ${ }^{\circledR}$, mBCA 515 Medical Body Composition Analyzer). Their inflammatory status was assessed with high-sensitivity C-reactive protein (hs-CRP), and insulin resistance with HOMA index. The inflammatory potential of their diet was assessed by the Dietary Inflammatory Index (DII®), based on 37 food parameters which derived from 123 -item food frequency questionnaire. A positive DII ${ }^{\circledR}$ designates a diet with pro-inflammatory potential, while negative DII ${ }^{\circledR}$ designates a diet with anti-inflammatory potential. Data and z-scores were analyzed with Statistica v13.3 (StatSoft Inc., Tulsa, USA) for baseline and study end parameters differences. Obtained results have shown that, at the end of study, the patients significantly reduced their body weight $(-3.3 \%, \mathrm{p}<0.001)$, BMI $(-3.2 \%, \mathrm{p}<0.001)$, waist circumference $(-1.9 \%, \mathrm{p}<0.001)$, total $(-3.3 \%, \mathrm{p}<0.001)$ and visceral body fat $(-10.4 \%, \mathrm{p}<0.001)$, total $(-3.1 \%, \mathrm{p}<0.001)$ and extracellular water $(-2.9 \%, \mathrm{p}<0.001)$. The patients also significantly reduced hs-CRP $(-37.7 \%, \mathrm{p}=0.0025)$. Although we observed noteworthy $-10.7 \%$ reduction of HOMA index, this finding did not reach statistical significance $(p=0.862)$. The inflammatory potential of patients' diet changed significantly from -0.45 to -1.73 on average $(p<0.001)$. With presented dietary intervention study it could be concluded that the change of the diet toward more anti-inflammatory milieu had significant influence on body weight, total and visceral body fat reduction. Consequently, those changes could have been involved in observed significant improvement of their inflammatory status. However, mild reduction of insulin resistance and rather small study group pointed out the need for further studies with more comprehensive dietary intervention.
\end{abstract}

\section{Conflict of Interest}

There is no conflict of interest. 